Case Report

\title{
A Case of an Acquired Factor VIII Inhibitor Complicated by Multiple Treatment-Related Opportunistic Infections and Review of the Literature
}

\author{
Anna L. Hutchinson, ${ }^{1}$ Yi Ling Tan, ${ }^{1}$ and Giselle Kidson-Gerber ${ }^{2}$ \\ ${ }^{1}$ Prince of Wales Hospital, Barker Street, Randwick, NSW 2031, Australia \\ ${ }^{2}$ Department of Medicine, University of New South Wales, Botany Street, Sydney, NSW 2052, Australia
}

Correspondence should be addressed to Anna L. Hutchinson; anna.ostberg@gmail.com

Received 15 October 2013; Accepted 26 November 2013

Academic Editors: S. Davidson and N. Hamerschlak

Copyright ( 92013 Anna L. Hutchinson et al. This is an open access article distributed under the Creative Commons Attribution License, which permits unrestricted use, distribution, and reproduction in any medium, provided the original work is properly cited.

\begin{abstract}
This case report describes a patient with an idiopathic acquired Factor VIII inhibitor and severe bleeding. She was treated with rituximab after failing first-line treatment with steroids and cyclophosphamide. Two months following rituximab treatment, our patient developed a succession of severe opportunistic infections requiring intensive care unit admission. Over a period of 12 weeks she required treatment for Pseudomonas aeruginosa septicaemia, herpes simplex gingivostomatitis and pharyngotonsillitis, clostridium difficile-related diarrhoea, systemic cytomegalovirus infection, pneumocystis jiroveci, and invasive pulmonary aspergillosis lung infections. After significant rehabilitation, the patient was finally discharged following a 5-month admission. This case highlights the complexity of balancing a life-threatening condition with the side effects of treatment. It also raises the issue of routine prophylaxis for immunosuppression in nonmalignant conditions, which will become a common dilemma with the expanding indications for rituximab use.
\end{abstract}

\section{Introduction}

The incidence of acquired inhibitors against FVIII is 1 to 4 per million/year in the nonhemophilic population [1]. In affected patients, the rate of severe bleeds is up to $90 \%$, with mortality rates between 8 and $22 \%$ [2]. This condition is generally diagnosed after detection of an isolated prolonged activated partial thromboplastin time (APTT), with failure to correct on mixing studies, and subsequent identification of reduced FVIII levels and presence of FVIII inhibitor. Most cases of acquired FVIII inhibitor are idiopathic, but up to $50 \%$ are associated with autoimmune diseases, malignancies, medications, or the postpartum period $[2,3]$.

Treatment of acute bleeding episodes is tailored according to inhibitor titre, site, and severity of bleeding. In high-titre patients, bypassing agents such as recombinant factor VIIa or FVIII inhibitor bypass activity (FEIBA) are indicated [1]. In patients with a low titre inhibitor (i.e., $<5$ Bethesda units or BU), plasma-derived or recombinant human FVIII can be used [1].

Current first-line treatment for eradication of FVIII inhibitor is oral corticosteroid [3,4]; this may be combined with cyclophosphamide [3]. Although combination with cyclophosphamide results in a greater remission rate than steroid alone, the increased rate of neutropenia-related infection means that the overall mortality rate is unchanged [4]. There is increasing evidence for the efficacy of rituximab (RTX) in those who fail first-line treatment or as firstline treatment for patients in whom corticosteroids and chemotherapeutic agents are contraindicated [3, 5-7]. RTX is a chimeric anti-CD20 monoclonal antibody widely used in the treatment of autoimmune disorders. It leads to the depletion of CD20+ B cells, which is hypothesised to interrupt autoantibody production. Berezné et al. report that RTX can be considered as first- or second-line treatment, either alone or in combination with cyclophosphamide [7]. Treatment 
of refractory FVIII inhibitor may also include intravenous immunoglobulin administration and immunoadsorption, particularly when bleeding cannot be controlled $[4,8]$.

\section{Case Study}

A 66-year-old woman with a background of type 2 diabetes mellitus was referred to the hematology service with bleeding after investigatory colonoscopy for symptomatic anemia. After colonoscopy she developed melena, hematuria, extensive subcutaneous hemorrhage, and a subsequent retroperitoneal hematoma. The severity of her bleeding required more than 30 packed red cell transfusions during her admission, FVIII concentrate, and tranexamic acid. There was no personal or family history of bruising or bleeding, and no underlying malignancy or autoimmune disorders were detected. HIV testing was negative.

The APTT was 79 seconds (reference range 25-37 seconds) with previously normal APTTs. Specific investigations demonstrated a strong FVIII inhibitor (234 BU) and residual FVIII activity of $<1 \%$ (reference range: 50-150\%). Initial management involved high-dose oral prednisone $50 \mathrm{mg}$ daily and cyclophosphamide $100 \mathrm{mg}$ daily. The prednisone was continued for 3 months and weaned to cessation over the fourth month. The cyclophosphamide was continued for 3 months and then ceased. No routine antimicrobial prophylaxis was concurrently given.

Three weeks following treatment initiation there was no improvement in APTT, FVIII inhibitor levels, or FVIII levels. Four cycles of RTX $375 \mathrm{mg} / \mathrm{m}^{2}$ weekly were initiated. Six weeks after commencement of RTX treatment, there was improvement of the APTT, FVIII inhibitor level, and FVIII levels (see Table 1). At 5 months after RTX treatment, the APTT and FVIII levels had normalised.

Two months after RTX treatment commenced and whilst receiving ongoing cyclophosphamide and prednisone, the patient required resuscitation for Pseudomonas aeruginosa septicemia. This was followed within a week by herpes simplex gingivostomatitis and pharyngotonsillitis and diarrhoea secondary to clostridium difficile infection. Pancytopenia developed with a neutrophil nadir of $0.9 \times 10^{9} / \mathrm{L}$.

Two weeks later, pneumocystis jiroveci and invasive pulmonary aspergillosis were diagnosed on bronchoscopy washings and brushings. Clinical cytomegalovirus (CMV) infection was confirmed by positive CMV nucleic acid testing of lung biopsy and serum and urine PCR. The prednisone dose was weaned and ceased. Cyclophosphamide was also ceased. Multiple intensive care unit admissions with respiratory support were necessary to manage these complications, and multiple courses of antibiotics and antifungals were required. After a five-month admission requiring significant rehabilitation, the patient was discharged. The factor VIII inhibitor remains in remission.

At presentation to hospital the patient was found to have hypogammaglobulinemia (IgG level of $4.75 \mathrm{mg} / \mathrm{dL}$, normal range $7-16 \mathrm{mg} / \mathrm{dL}$ ). She received 3 doses of intravenous immunoglobulin 4 weeks apart. Interestingly, she was hypogammaglobulinemic prior to RTX and remains
TABLE 1: APTT, FVIII level, and inhibitor level over time.

\begin{tabular}{lccc}
\hline Time point & $\begin{array}{c}\text { APTT (sec) } \\
{[\text { RR 25-37 sec] }}\end{array}$ & $\begin{array}{c}\text { FVIII inhibitor } \\
\text { level (BU) } \\
\text { [normal: 0 BU] }\end{array}$ & $\begin{array}{c}\text { FVIII level (\%) } \\
\text { [RR 50-150\%] }\end{array}$ \\
\hline Presentation & 83 & 234 & $<1 \%$ \\
3 weeks & 77 & 509 & $<1 \%$ \\
9 weeks & 36 & 118 & $22 \%$ \\
5 months & 26.7 & Not tested & $187 \%$ \\
\hline
\end{tabular}

RR: reference range.

hypogammaglobulinemic 3 years after this episode. The cause of this is unknown; her $\operatorname{IgA}, \operatorname{IgG}$, and $\operatorname{IgM}$ are all below the reference range.

The patient was taking metformin 1 gram twice daily and gliclazide $160 \mathrm{mg}$ twice daily at the time of admission. Her blood sugar level (BSL) control was poor prior to treatment with prednisone (range $7-20 \mathrm{mmol} / \mathrm{L}$ ) and deteriorated further with steroids (range $13-24 \mathrm{mmol} / \mathrm{L}$ ). Despite additional sliding-scale short- and intermediate-acting insulin, her BSL control remained unsatisfactory throughout her admission (range 4-20 $\mathrm{mmol} / \mathrm{L}$ ).

\section{Discussion}

This case highlights the complexity of balancing a potentially life-threatening condition with the side effects of therapy. Disease control was only achieved after administering second-line therapy, however at the cost of more infections. Severe immunosuppression is an accepted risk in the management of malignancy and organ transplantation, but less so with autoimmune conditions. Our patient's immunosuppression led to successive severe infections: over a period of 12 weeks she suffered from clinically significant infections with Pseudomonas aeruginosa septicaemia, herpes simplex, clostridium difficile, pneumocystis jiroveci, invasive pulmonary aspergillosis, and systemic CMV.

Published case reports document the efficacy of RTX in the treatment of refractory FVIII inhibitors $[1,9,10]$. Wiestner et al. treated 4 consecutive patients with acquired FVIII inhibitor with RTX: 3 in combination with corticosteroids and one with RTX as monotherapy. In all cases, the inhibitor resolved within several weeks and the patients remained in remission during followup of 7 to 12 months [9]. The majority of current literature involves using RTX as second-line treatment and as combination rather than monotherapy $[1,5,11]$. RTX may be adequate to effectively treat low inhibitor level patients, but those with high level inhibitors $(>100 \mathrm{BU} / \mathrm{mL})$ generally require the addition of cyclophosphamide and corticosteroids [11].

Since the advent of such agents as FEIBA, recombinant factor VIIa, and desmopressin, the complications of treatment for acquired FVIII inhibitor have become a greater cause of morbidity and mortality than the risk of bleeding itself $[8,11]$. Case reports in the literature document the sideeffects of treatment; however, none describe complications as severe as outlined in this case. We support the efficacy 
of RTX; however, we wish to caution against the possible complications. We propose that the addition of RTX to an established immunosuppressant regime of prednisone and cyclophosphamide played a significant role in the development of successive life-threatening and opportunistic infections, whilst acknowledging contribution by poorlycontrolled diabetes and hypogammaglobulinemia. A causal relationship cannot be confirmed, however, due to the administration of additional immunosuppressive therapy.

Several case reports regarding the use of RTX have highlighted its potential to cause cytopenias, particularly when given in combination with other chemotherapeutics [12]. In 72 patients with non-Hodgkin's lymphoma treated with RTX, $30 \%$ developed neutropenia [12]. Of these 21 patients, $19 \%$ suffered a major infection. These infections included $P$. carinii pneumonia, CMV reactivation, mycobacterial pneumonia, CMV pneumonitis, and bacterial pneumonia [12]. Neutropenia typically develops greater than 4 weeks following RTX treatment, in keeping with our patient's presentation [13], and resolves after an average of 11 weeks [12].

There have been multiple reports documenting the potential for the development of opportunistic infections in RTXtreated patients, in particular viral infections. In a case series of 64 patients with severe viral infections following combination RTX and chemotherapy, the range of viruses encountered included hepatitis B (HBV, $n=25)$, CMV infection $(n=15)$, varicella zoster virus (VZV, $n=6)$, and others including herpes simplex virus (HSV) [14]. One-third of non-HBV infections were fatal [14].

A study of 77 patients who received RTX after renaltransplant found that $45 \%$ developed infections: bacterial, viral, and fungal infections were seen in $36 \%, 18 \%$, and $17 \%$ of the cohort, respectively [15]. A control group of 902 patients who did not receive RTX had an infection rate similar to that of the RTX group; however, the mortality rate from infection was significantly lower in this group (1.6\%) compared with that in the RTX group (9.1\%). Infections occurred an average of 3 months after RTX treatment commenced and the most common were septic shock, Escherichia coli septicemia, pneumonia, pyelonephritis, CMV, HSV, HBV reactivation, candidemia, aspergillosis, pneumocystosis, and cryptococcal meningitis [15]. A review of RTX use in autoimmune diseases (excluding rheumatoid arthritis) across 25 studies involving 389 patients shows the incidence of serious infections to range from 3 to $33 \%$ [16]. The mortality rate from infectious complications was $9 \%$ overall [16].

RTX is known to cause neutropenia [12, 17], hypogammaglobulinemia [18], and thrombocytopenia [12] and to increase the risk of infection [18]. Neutropenia usually occurs greater than 4 weeks after treatment commencement; the median time to development is 10 weeks (range 3-23) and average duration 11 weeks (range 1-23+) [12]. Late onset neutropenia (LON) has been seen in RTX monotherapy but is more common when combined with chemotherapy; LON occurs in $7-30 \%$ of RTX-treated patients $[12,13,17]$. The pathogenesis of LON remains uncertain; however, bone marrow biopsy in these patients reveals maturation arrest [17]. The development of neutropenia is strongly associated with the number of RTX doses [12].
Hypogammaglobulinemia occurs with increasing frequency the more cycles of RTX are given and is seen in patients treated for autoimmunity as well as for malignancy [19]. Of interest, the different subclasses are affected to different extents. In a study of over 1000 patients, IgM levels fell by $10 \%$ after a single course, IgA by $4 \%$, and IgG by $2 \%$ [19] . A review of the literature did not locate any articles linking poorly controlled diabetes or hypogammaglobulinemia to the list of infections suffered by this patient.

\section{Teaching Points}

We felt it was important to document this case to alert clinicians to the potential adverse effects of immunosuppression for nonmalignant conditions. RTX-treated patients should be closely monitored and promptly treated for opportunistic infections. Clinicians should be especially alert in patients with additional immune dysregulation and some patients may benefit from routine prophylaxis against viruses, bacteria, and fungi. We did not give antimicrobial prophylaxis to this patient; however, in retrospect it would have been appropriate. The combination of RTX with other immunosuppressive treatment rather than chemotherapy may have different ramifications, and this will become apparent with the expanding indications for RTX use.

\section{References}

[1] M. Franchini and G. Lippi, "Acquired factor VIII inhibitors," Blood, vol. 112, no. 2, pp. 250-255, 2008.

[2] D. Green and K. Lechner, "A survey of 215 non-hemophilic patients with inhibitors to factor VIII," Thrombosis and Haemostasis, vol. 45, no. 3, pp. 200-203, 1981.

[3] J. Muzaffar, L. Katragadda, S. Haider, A. Javed, E. Anaissie, and S. Usmani, "Rituximab and intravenous immunoglobulin (IVIG) for the management of acquired factor VIII inhibitor in multiple myeloma: case report and review of literature," International Journal of Hematology, vol. 95, no. 1, pp. 102-106, 2012.

[4] B. Barnett, R. Kruse-Jarres, and C. A. Leissinger, "Current management of acquired factor VIII inhibitors," Current Opinion in Hematology, vol. 15, no. 5, pp. 451-455, 2008.

[5] J. J. Field, T. S. Fenske, and M. A. Blinder, "Rituximab for the treatment of patients with very high-titre acquired factor VIII inhibitors refractory to conventional chemotherapy," Haemophilia, vol. 13, no. 1, pp. 46-50, 2007.

[6] W. R. Sperr, K. Lechner, and I. Pabinger, "Rituximab for the treatment of acquired antibodies to factor VIII," Haematologica, vol. 92, no. 1, pp. 66-71, 2007.

[7] A. Berezné, N. Stieltjes, V. Le-Guern et al., "Rituximab alone or in association with corticosteroids in the treatment of acquired factor VIII inhibitors: report of two cases," Transfusion Medicine, vol. 16, no. 3, pp. 209-212, 2006.

[8] R. S. Schwartz, D. A. Gabriel, L. M. Aledort, D. Green, and C. M. Kessler, "A prospective study of treatment of acquired (autoimmune) factor VIII inhibitors with high-dose intravenous gammaglobulin," Blood, vol. 86, no. 2, pp. 797-804, 1995.

[9] A. Wiestner, H. J. Cho, A. S. Asch et al., "Rituximab in the treatment of acquired factor VIII inhibitors," Blood, vol. 100, no. 9, pp. 3426-3428, 2002. 
[10] M. Wermke, M. von Bonin, S. Gehrisch, G. Siegert, G. Ehninger, and U. Platzbecker, "Successful eradication of acquired factorVIII-inhibitor using single low-dose rituximab," Haematologica, vol. 95, no. 3, pp. 521-522, 2010.

[11] Y. Alvarado, X. Yao, C. Jumper, F. Hardwicke, N. D'Cunha, and E. Cobos, "Acquired hemophilia: a case report of 2 patients with acquired factor VIII inhibitor treated with rituximab plus a short course of steroid and review of the literature," Clinical and Applied Thrombosis/Hemostasis, vol. 13, no. 4, pp. 443-448, 2007.

[12] C. Cattaneo, P. Spedini, S. Casari et al., "Delayed-onset peripheral blood cytopenia after rituximab: frequency and risk factor assessment in a consecutive series of 77 treatments," Leukemia \& Lymphoma, vol. 47, no. 6, pp. 1013-1017, 2006.

[13] E. Nitta, K. Izutsu, T. Sato et al., "A high incidence of late-onset neutropenia following rituximab-containing chemotherapy as a primary treatment of CD20-positive B-cell lymphoma: a singleinstitution study," Annals of Oncology, vol. 18, no. 2, pp. 364-369, 2007.

[14] S. Aksoy, H. Harputluoglu, S. Kilickap et al., "Rituximab-related viral infections in lymphoma patients," Leukemia \& Lymphoma, vol. 48, no. 7, pp. 1307-1312, 2007.

[15] N. Kamara, O. Miliotoa, B. Puissant-Lubranoc et al., "Incidence and predictive factors for infectious disease after rituximab therapy in kidney-transplant patients," The American Journal of Transplantation, vol. 10, no. 1, pp. 89-98, 2010.

[16] H. M. Gürcan, D. B. Keskin, J. N. H. Stern, M. A. Nitzberg, H. Shekhani, and A. R. Ahmed, "A review of the current use of rituximab in autoimmune diseases," International Immunopharmacology, vol. 9, no. 1, pp. 10-25, 2009.

[17] D. Tesfa, T. Gelius, B. Sander et al., "Late-onset neutropenia associated with rituximab therapy: evidence for a maturation arrest at the (pro)myelocyte stage of granulopoiesis," Medical Oncology, vol. 25, no. 4, pp. 374-379, 2008.

[18] N. Cooper and D. M. Arnold, "The effect of rituximab on humoral and cell mediated immunity and infection in the treatment of autoimmune diseases," British Journal of Haematology, vol. 149, no. 1, pp. 3-13, 2010.

[19] E. Keystone, R. Fleischmann, P. Emery et al., "Safety and efficacy of additional courses of rituximab in patients with active rheumatoid arthritis: an open-label extension analysis," Arthritis \& Rheumatism, vol. 56, no. 12, pp. 3896-3908, 2007. 


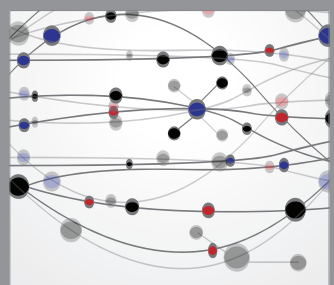

The Scientific World Journal
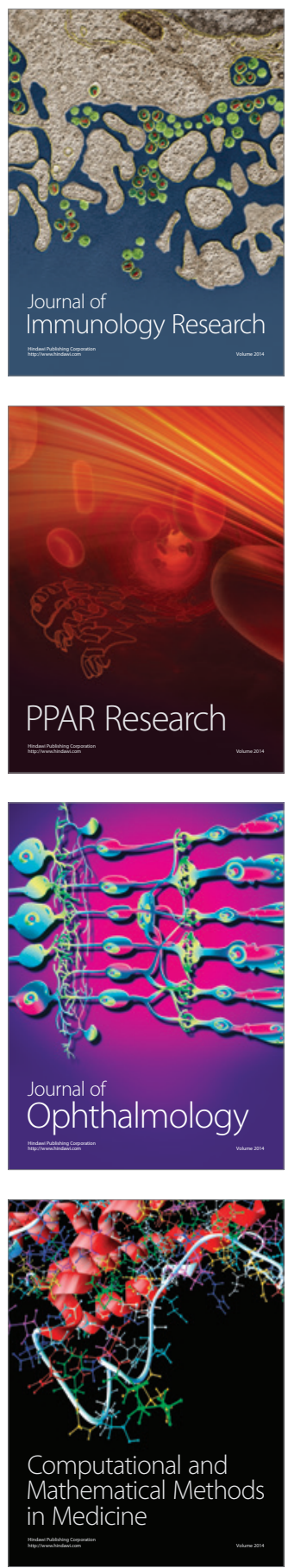

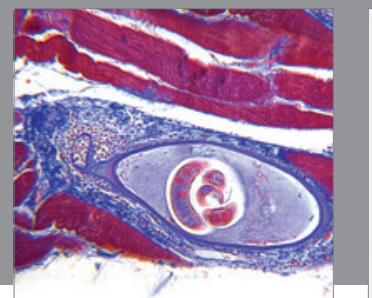

Gastroenterology

Research and Practice
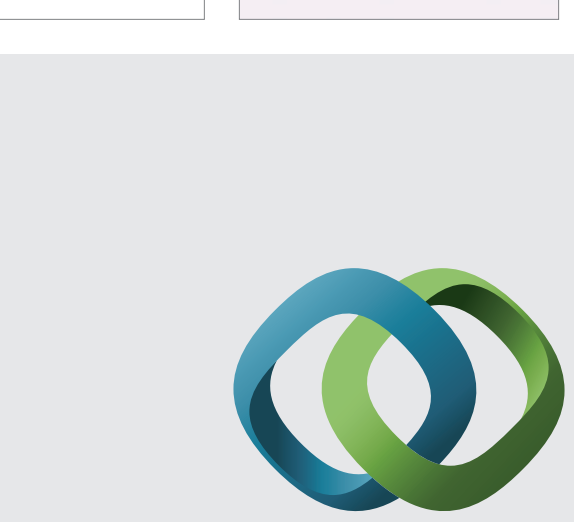

\section{Hindawi}

Submit your manuscripts at

http://www.hindawi.com
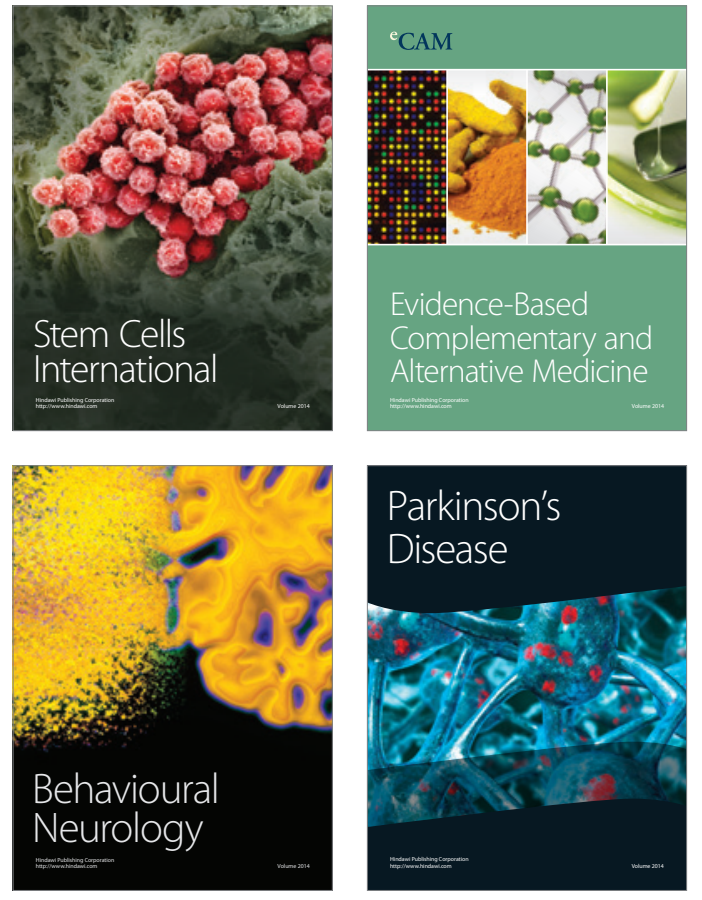
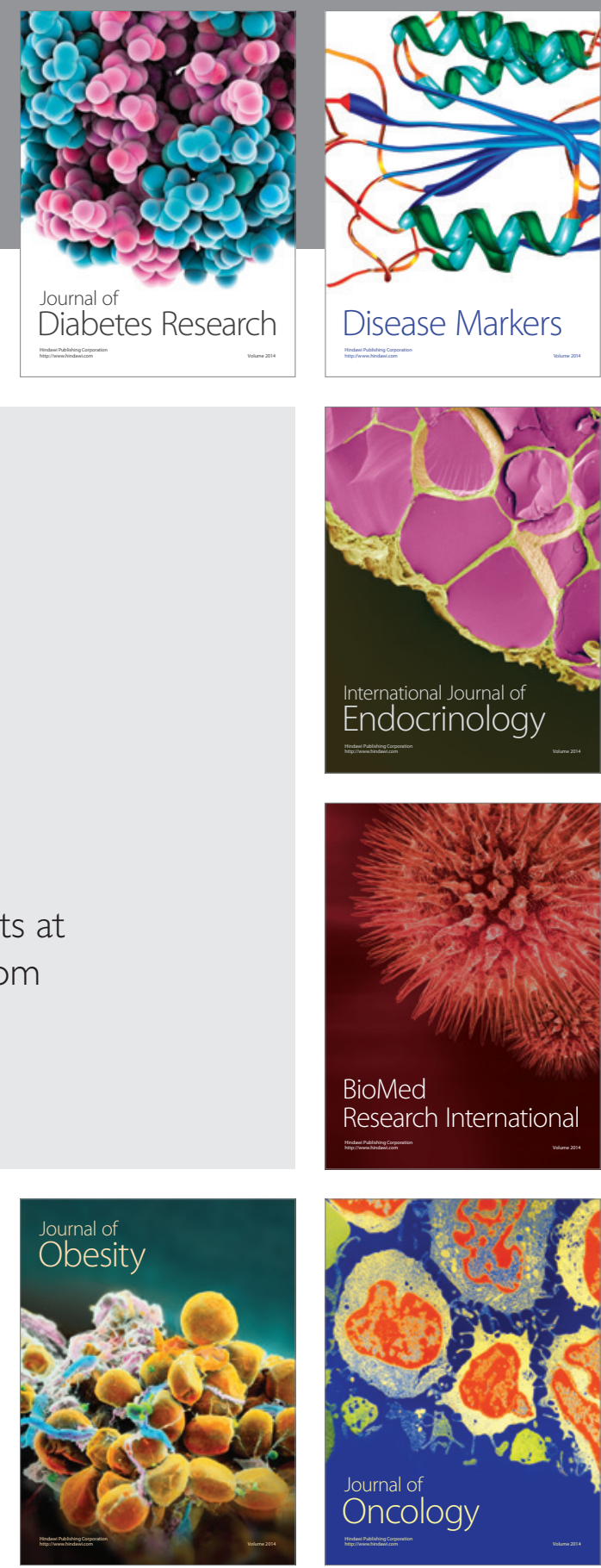

Disease Markers
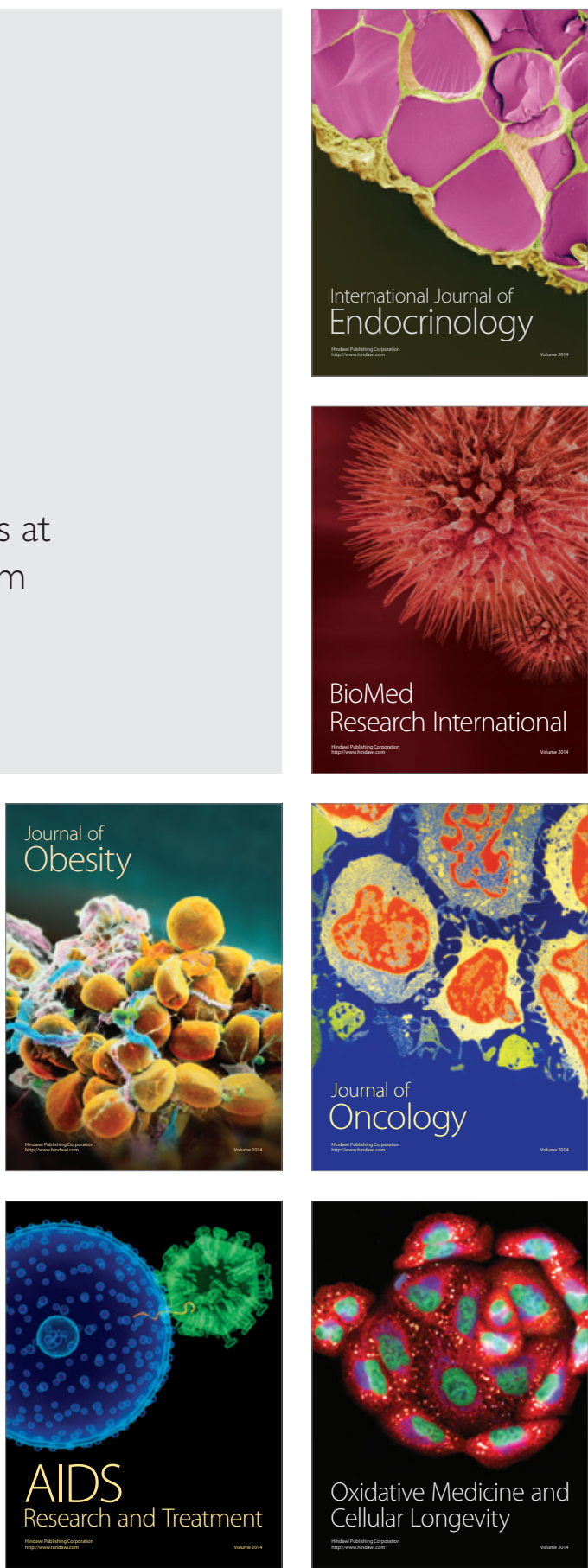